\title{
SENSORS AND BOUNDARY STATE RECONSTRUCTION OF HYPERBOLIC SYSTEMS
}

\author{
EL HASSAN ZERRIK * , HAMID BOURRAY **, SAMIR BEN HADID *** \\ ${ }^{*}$ MACS Group, Faculty of Sciences \\ University of Moulay Ismaill, Bni Mhamed 4011, Meknes, Morocco \\ e-mail:zerrik3eyahoo.fr \\ ** MACS Group, Faculty of Poly Disciplinary \\ University of Moulay Ismaïl, Boutalamine I, Errachidia, Morocco \\ e-mail: hbourrayh@yahoo.fr \\ *** Department of Mathematics, Faculty of Exact Sciences \\ Mentouri University, Constantine, Algeria \\ e-mail: ihebmaths@yahoo.fr
}

\begin{abstract}
This paper deals with the problem of regional observability of hyperbolic systems in the case where the subregion of interest is a boundary part of the system evolution domain. We give a definition and establish characterizations in connection with the sensor structure. Then we show that it is possible to reconstruct the system state on a subregion of the boundary. The developed approach, based on the Hilbert uniqueness method (Lions, 1988), leads to a reconstruction algorithm. The obtained results are illustrated with numerical examples and simulations.
\end{abstract}

Keywords: distributed systems, hyperbolic systems, regional observability, boundary reconstruction, strategic sensor.

\section{Introduction}

The study of dynamical spatiotemporal systems has generated wide literature with applications in fields such as ecology, pollution control, population dynamics as well as many others. A wide portion of the literature is devoted to the problem of the analysis and control partial differential equations, and many notions have been studied and explored. Numerous works have been devoted to the observation problem in the whole domain, see (Gilliam and Martin, 1988; Kobayashi, 1980). But some delicate problems need to be studied only in some subregion of the system evolution domain. This is the subject of the regional control theory of distributed parameter systems (DPSs), which was pioneered by El Jai and his co-workers since the 1990s and consists in studying notions related to control and observation only on a subregion of the system evolution domain. The reader may find interesting developments of these topics for parabolic and hyperbolic systems, see (Amouroux et al., 1994), when the subregion is interior to the system domain; one also finds examples for a system which is not observable (controllable) within the whole domain $\Omega$ but observable (controllable) in a subregion $\omega \subset \Omega$. An extension of these results to a boundary subregion for parabolic systems was then discussed in (Zerrik et al., 2002).

There are many applications of these notions and an interesting one may be the problem of determining laminar boundary flux conditions developed in a steady-state by a vertical heated plate and consists of the study of the thermal transfer by natural convection generated by a uniformly heated plate located in a small enclosure. Inside that enclosure, differences in the wall surface produce natural convection movements. The heat exchanger maintains a prescribed temperature on the back face of the plate by means of hot water circulation. All the faces of this active wall are insulated except for the front face. The objective is to find the unknown boundary convective condition on a part of the front face of the active plate using measurements given by internal thermocouples, see (Aparron, 1963) for more details.

There is an extensive literature on the exact and approximate regional observability problem for linear pa- 
rabolic systems, but very little has been done for hyperbolic ones. Recently, regional observability for hyperbolic systems has been introduced in the internal case and the developed theory leads to interesting results performed through numerical examples and simulations (Zerrik et al., 2007).

Here we are interested in the regional observability of hyperbolic systems where the subregion target is a part of the boundary of the system evolution domain. We establish results which are extensions of those given in (Zerrik et al., 2002) to a class of hyperbolic systems. This is the aim of this paper, which is organized as follows: In Section 2 we introduce definitions and properties of regional observability and show that regional observability implies boundary one. In Section 3 we characterize the sensor which ensures regional boundary observability. In Section 4 we give two approaches for regional reconstruction: the first one is direct and based on pseudoinverse techniques and the second one uses an extension of the Hilbert uniqueness method. In the last section we give an example of hyperbolic systems in a two-dimensional case which illustrates the obtained results through numerical simulations.

\section{Regional observability}

Let $\Omega$ be an open bounded subset of $\mathbb{R}^{n}$, with a boundary $\partial \Omega$ which is regular enough. For $T>0$, we set $Q=\Omega \times] 0, T[, \Sigma=\partial \Omega \times] 0, T[$ and we consider a system described by the equation

$$
\begin{cases}\frac{\partial^{2} y}{\partial t^{2}}(x, t)+\mathcal{A} y(x, t)=0 & \text { in } Q, \\ y(x, 0)=y^{0}(x), \quad \frac{\partial y}{\partial t}(x, 0)=y^{1}(x) & \text { in } \Omega, \\ \frac{\partial y}{\partial \nu_{\mathcal{A}}}(\zeta, t)=0 & \text { on } \Sigma,\end{cases}
$$

where $\mathcal{A}$ is the elliptic differential operator of the second order given by

$$
\mathcal{A}=-\sum_{i, j=1}^{n} \frac{\partial}{\partial x_{i}}\left(a_{i j} \frac{\partial}{\partial x_{j}}\right)
$$

with domain

$$
\mathcal{D}(\mathcal{A})=\left\{\varphi \in H^{2}(\Omega), \frac{\partial \varphi}{\partial \nu_{\mathcal{A}}}=0 \quad \text { on } \partial \Omega\right\} .
$$

We assume that

$$
a_{i j}=a_{j i} \in C^{1}(\Omega)
$$

and there exists $\alpha>0$ such that

$$
\sum_{i, j=1}^{n} a_{i j} \zeta_{i} \zeta_{j} \geq \alpha \sum_{j=1}^{n}\left|\zeta_{j}\right|^{2}, \quad \forall \zeta=\left(\zeta_{1}, \ldots, \zeta_{n}\right) \in \mathbb{R}^{n},
$$

where

$$
\frac{\partial y}{\partial \nu_{\mathcal{A}}}=\sum_{i, j=1}^{n} a_{i j} \frac{\partial y}{\partial x_{j}} \eta_{i}
$$

is the conormal derivative of the operator $\mathcal{A}$, and $\eta_{i}$ stands for the $i$-th component of the conormal $\eta$ to $\partial \Omega$.

We consider the state space $\mathcal{F}=H^{2}(\Omega) \times H^{1}(\Omega)$ and $\mathcal{O}=L^{2}\left(0, T ; \mathbb{R}^{q}\right)$ as the observation space. The system (1) is augmented with the output

$$
z(t)=C y(t)
$$

where $C: H^{2}(\Omega) \longrightarrow \mathbb{R}^{q}$ is the observation operator, $q$ indicates the number of the sensors considered, see (El Jai and Pritchard, 1988).

Let the operator

$$
A=\left(\begin{array}{ll}
0 & I \\
\mathcal{A} & 0
\end{array}\right)
$$

be defined by

$$
A\left(z_{1}, z_{2}\right)=\left(z_{2}, \mathcal{A} z_{1}\right)
$$

for all

$$
\left(z_{1}, z_{2}\right) \in D(A)=D(\mathcal{A}) \times H^{1}(\Omega) .
$$

This operator generates a semigroup $(S(t))_{t \geq 0}$.

Denoting $\bar{y}=(y, \partial y / \partial t)$, the system (1) may be written in the following form:

$$
\left\{\begin{array}{l}
\frac{\partial \bar{y}}{\partial t}=A \bar{y} \text { in } \quad Q \\
\bar{y}(0)=\bar{y}^{0} \quad \text { in } \quad \Omega
\end{array}\right.
$$

where $\bar{y}^{0}=\left(y^{0}, y^{1}\right)$ and the output function (2) takes the form

$$
\bar{z}(t)=\mathcal{C} \bar{y}(t)
$$

for $\mathcal{C}=(C, 0)$. Then the system (3) admits a unique solution given by

$$
\bar{y}(t)=S(t) \bar{y}^{0} .
$$

Using (4), we obtain

$$
\bar{z}(t)=\left(K \bar{y}^{0}\right)(t)
$$

where $K: H^{2}(\Omega) \times H^{1}(\Omega) \longrightarrow \mathcal{O}=L^{2}\left(0, T ; \mathbb{R}^{q}\right)$ given by $K y=\mathcal{C} S()$.$y .$

Let $w_{m}$ be the basis of eigenfunctions of the operator $A$ and $\lambda_{m}$ be the associated eigenvalues with multiplicities $r_{m}$. Then the semigroup $(S(t))_{t \geq 0}$ generated by the 
operator $A$ is given by

$$
\begin{aligned}
& S(t)\left(y^{1}, y^{2}\right)
\end{aligned}
$$

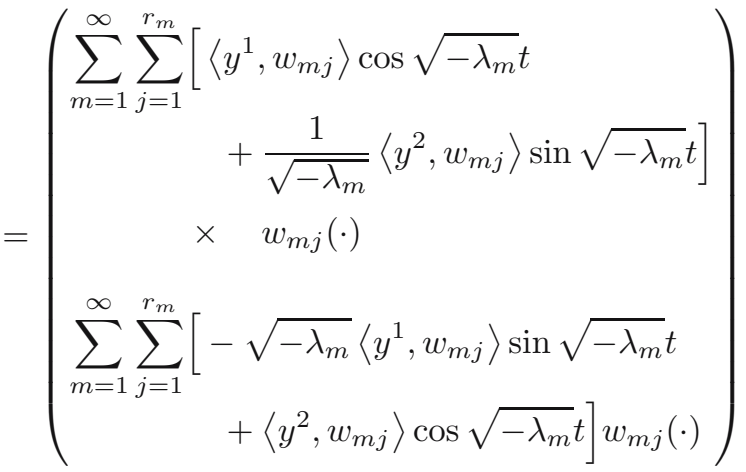

Consider a regular boundary portion $\Gamma$ of $\partial \Omega$ with a positive measure and let $\omega$ an open part of $\Omega$ with a regular boundary $\partial \omega$ such that $\Gamma \subset \partial \Omega \cap \partial \omega$. Then one can consider restriction operators defined by

$$
\begin{aligned}
\chi_{\omega}: H^{2}(\Omega) \times H^{1}(\Omega) & \longrightarrow H^{2}(\omega) \times H^{1}(\omega) \\
\left(y^{1}, y^{2}\right) & \longrightarrow \chi_{\omega}\left(y^{1}, y^{2}\right) \\
& =\left.\left(y^{1}, y^{2}\right)\right|_{\omega}, \\
\chi_{\Gamma}: H^{3 / 2}(\partial \Omega) \times H^{1 / 2}(\partial \Omega) & \longrightarrow H^{3 / 2}(\Gamma) \times H^{1 / 2}(\Gamma) \\
\left(y^{1}, y^{2}\right) & \longrightarrow \chi_{\Gamma}\left(y^{1}, y^{2}\right) \\
& =\left.\left(y^{1}, y^{2}\right)\right|_{\Gamma},
\end{aligned}
$$

and the trace operator

$$
\gamma_{0}: H^{2}(\Omega) \times H^{1}(\Omega) \longrightarrow H^{3 / 2}(\partial \Omega) \times H^{1 / 2}(\partial \Omega),
$$

where $\gamma_{0}^{*}, \chi_{\Gamma}^{*}$ and $\chi_{\omega}^{*}$ denote respectively the adjoints of $\gamma_{0}, \chi_{\Gamma}$ and $\chi_{\omega}$. We denote by $K^{*}$ the adjoint of $K$ and obtain what follows.

\section{Definition 1.}

1. The system (1) augmented with (2) is said to be exactly (resp. weakly) observable in $\omega$ if $\operatorname{Im} \chi_{\omega} K^{*}=$ $H^{2}(\omega) \times H^{1}(\omega)\left(\right.$ resp. $\overline{\operatorname{Im} \chi_{\omega} K^{*}}=H^{2}(\omega) \times$ $\left.H^{1}(\omega)\right)$.

2. The system (1) augmented with (2) is said to be exactly (resp. weakly) observable on $\Gamma$ if $\operatorname{Im} \chi_{\Gamma} \gamma_{0} K^{*}=$ $H^{3 / 2}(\Gamma) \times H^{1 / 2}(\Gamma)\left(\operatorname{resp} \overline{\operatorname{Im} \chi_{\Gamma} \gamma_{0} K^{*}}=H^{3 / 2}(\Gamma) \times\right.$ $\left.H^{1 / 2}(\Gamma)\right)$.

Remark 1. If the system is exactly (resp. approximately) observable in $\omega$, then it is exactly (resp. approximately) observable in every subset $\omega_{1} \subset \omega$.

Problem. Given the system (1) augmented with the output (2), is it possible to reconstruct the initial state of the system (1) on $\Gamma$ ?

From the above definitions we have the following.
Proposition 1. If the system (11) augmented with (2) is exactly (resp. weakly) observable in $\omega$, then it is exactly (resp. weakly) observable on $\Gamma$.

Proof. (Part 1) Let us show that if the system (1) is exactly observable in $\omega$ then it is exactly observable on $\Gamma$. For this purpose, it is sufficient to show that

$$
H^{3 / 2}(\Gamma) \times H^{1 / 2}(\Gamma) \subset \operatorname{Im} \chi_{\Gamma} \gamma_{0} K^{*} .
$$

Let $\left(y^{1}, y^{2}\right) \in H^{3 / 2}(\Gamma) \times H^{1 / 2}(\Gamma)$ and let $\left(\tilde{y}^{1}, \tilde{y}^{2}\right)$ be its extension to $H^{3 / 2}(\partial \Omega) \times H^{1 / 2}(\partial \Omega)$.

Applying the trace theorem, there exists a continuous harmonic operator

$$
\mathcal{R}: H^{3 / 2}(\partial \Omega) \times H^{1 / 2}(\partial \Omega) \longrightarrow H^{2}(\Omega) \times H^{1}(\Omega)
$$

such that

$$
\gamma_{0} \mathcal{R}\left(\tilde{y}^{1}, \tilde{y}^{2}\right)=\left(\tilde{y}^{1}, \tilde{y}^{2}\right)
$$

which yields

$$
\chi_{\omega} \mathcal{R}\left(\tilde{y}^{1}, \tilde{y}^{2}\right) \in H^{2}(\omega) \times H^{1}(\omega) .
$$

Since the system (1) is exactly observable in $\omega$, there exists $z_{1} \in \mathcal{O}$ such that

$$
\chi_{\omega} K^{*} z_{1}=\chi_{\omega} \mathcal{R}\left(\tilde{y}^{1}, \tilde{y}^{2}\right)
$$

and then

$$
\gamma_{0}\left(\chi_{\omega}^{*} \chi_{\omega} K^{*} z_{1}\right)=\gamma_{0}\left(\chi_{\omega}^{*} \chi_{\omega} \mathcal{R}\left(\tilde{y}^{1}, \tilde{y}^{2}\right)\right) .
$$

Thus

$$
\begin{aligned}
\chi_{\Gamma}\left(\gamma_{0}\left(\chi_{\omega}^{*} \chi_{\omega} K^{*} z_{1}\right)\right) & =\chi_{\Gamma}\left(\gamma_{0}\left(\chi_{\omega}^{*} \chi_{\omega} \mathcal{R}\left(\tilde{y}^{1}, \tilde{y}^{2}\right)\right)\right. \\
& =\left(y^{1}, y^{2}\right) .
\end{aligned}
$$

Using the fact that

$$
\chi_{\Gamma}\left(\gamma_{0}\left(\chi_{\omega}^{*} \chi_{\omega} K^{*} z_{1}\right)\right)=\chi_{\Gamma}\left(\gamma_{0}\left(K^{*} z_{1}\right)\right),
$$

we have

$$
\chi_{\Gamma}\left(\gamma_{0}\left(K^{*} z_{1}\right)\right)=\left(y^{1}, y^{2}\right)
$$

which means that the system (1)-(2) is exactly observable on $\Gamma$.

(Part 2) We must now show that

$$
\begin{aligned}
\forall \varepsilon>0, \forall\left(y^{1}, y^{2}\right) & \in H^{3 / 2}(\Gamma) \times H^{1 / 2}(\Gamma), \exists z_{1} \in \mathcal{O} \\
& \left\|\chi_{\Gamma}\left(\gamma_{0}\left(K^{*} z_{1}\right)\right)-\left(y^{1}, y^{2}\right)\right\|<\varepsilon
\end{aligned}
$$

Let $\left(y^{1}, y^{2}\right) \in H^{3 / 2}(\Gamma) \times H^{1 / 2}(\Gamma)$ and $\left(\tilde{y}^{1}, \tilde{y}^{2}\right)$ be its extension to $H^{3 / 2}(\partial \Omega) \times H^{1 / 2}(\partial \Omega)$. By the trace theorem, there exists $\mathcal{R}\left(\tilde{y}^{1}, \tilde{y}^{2}\right) \in H^{2}(\Omega) \times H^{1}(\Omega)$ such that

$$
\gamma_{0}\left(\mathcal{R}\left(\tilde{y}^{1}, \tilde{y}^{2}\right)\right)=\left(\tilde{y}^{1}, \tilde{y}^{2}\right)
$$

Since

$$
\chi_{\omega}\left(\mathcal{R}\left(\tilde{y}^{1}, \tilde{y}^{2}\right)\right) \in H^{2}(\omega) \times H^{1}(\omega)
$$


and the system (1)-(2) is weakly observable in $\omega$, we have

$$
\begin{aligned}
& \forall \varepsilon>0, \exists z_{1} \in \mathcal{O}, \\
& \left.\qquad \chi_{\omega}\left(K^{*} z_{1}\right)\right)-\chi_{\omega}\left(\mathcal{R}\left(\tilde{y}^{1}, \tilde{y}^{2}\right)\right) \|<\varepsilon
\end{aligned}
$$

By the continuity of the trace map $\gamma$ we have

$$
\begin{aligned}
& \left\|\gamma_{0}\left(\chi_{\omega}^{*} \chi_{\omega}\left(K^{*} z_{1}\right)-\chi_{\omega}^{*} \chi_{\omega}\left(\mathcal{R}\left(\tilde{y}^{1}, \tilde{y}^{2}\right)\right)\right)\right\| \\
& \leq\left\|\chi_{\omega}^{*} \chi_{\omega}\left(K^{*} z_{1}\right)-\chi_{\omega}^{*} \chi_{\omega}\left(\mathcal{R}\left(\tilde{y}^{1}, \tilde{y}^{2}\right)\right)\right\|_{H^{2}(\Omega) \times H^{1}(\Omega)} \\
& =\left\|\chi_{\omega}\left(K^{*} z_{1}\right)-\chi_{\omega}\left(\mathcal{R}\left(\tilde{y}^{1}, \tilde{y}^{2}\right)\right)\right\|_{H^{2}(\omega) \times H^{1}(\omega)}
\end{aligned}
$$

and

$$
\begin{aligned}
& \left\|\chi_{\Gamma} \gamma_{0}\left(\chi_{\omega}^{*} \chi_{\omega}\left(K^{*} z_{1}\right)-\chi_{\omega}^{*} \chi_{\omega} \mathcal{R}\left(\tilde{y}^{1}, \tilde{y}^{2}\right)\right)\right\| \\
& \quad \leq\left\|\gamma_{0}\left(\chi_{\omega}^{*} \chi_{\omega}\left(K^{*} z_{1}\right)-\chi_{\omega}^{*} \chi_{\omega}\left(\mathcal{R}\left(\tilde{y}^{1}, \tilde{y}^{2}\right)\right)\right)\right\|,
\end{aligned}
$$

but

$$
\begin{gathered}
\chi_{\Gamma} \gamma_{0}\left(\chi_{\omega}^{*} \chi_{\Gamma} K^{*} z_{1}-\chi_{\omega}^{*} \chi_{\omega} \mathcal{R}\left(\tilde{y}^{1}, \tilde{y}^{2}\right)\right) \\
=\chi_{\Gamma} \gamma_{0}\left(K^{*} z_{1}-\mathcal{R}\left(\tilde{y}^{1}, \tilde{y}^{2}\right)\right) \\
=\chi_{\Gamma} \gamma_{0} K^{*} z_{1}-\left(y^{1}, y^{2}\right)
\end{gathered}
$$

which gives

$$
\left\|\chi_{\Gamma} \gamma_{0} K^{*} z_{1}-\left(y^{1}, y^{2}\right)\right\| \leqslant \varepsilon .
$$

Then the system (1) is weakly observable on $\Gamma$.

Proposition 2. We have the equivalence between the following statements:

1. The system (1)-(2) is exactly observable on $\Gamma$.

2. There exists $c>0$ such that

$$
\begin{aligned}
\|y\|_{H^{\frac{3}{2}}(\Gamma) \times H^{\frac{1}{2}}(\Gamma)} & \leq c\left\|H^{*} y\right\|_{\mathcal{O}}, \\
\forall y & =\left(y_{1}, y_{2}\right) \in H^{\frac{3}{2}}(\Gamma) \times H^{\frac{1}{2}}(\Gamma),
\end{aligned}
$$

where $H=\chi_{\Gamma} \gamma_{0} K^{*}$.

Proof. The proof uses the following general results (see (Avdonin and Ivanov, 1978)). Let $E, F$, and $G$, be reflexive Banach spaces and $f \in \mathcal{L}(E, G), g \in \mathcal{L}(F, G)$. Then we have the equivalence between the statements below:

1. $\operatorname{Im}(f) \subset \operatorname{Im}(g)$.

2. There exists $c>0$ such that $\left\|f^{*} y\right\|_{E^{*}} \leq\left\|g^{*} y\right\|_{F^{*}}$.

We set $E=G=H^{\frac{3}{2}}(\Gamma) \times H^{\frac{1}{2}}(\Gamma), F=\mathcal{O}, f=$ $I d_{H^{\frac{3}{2}}(\Gamma) \times H^{\frac{1}{2}}(\Gamma)}$ and $g=H$, and obtain the inequality (8).

\section{Characterization of $\Gamma$-strategic sensors}

In this section, we shall characterize sensors which ensure approximate regional boundary observability in a portion $\Gamma$ of the boundary $\partial \Omega$. Let us reconsider the system (1) with measurements given by

$$
\left.z(t)=\left(z_{1}(t), \ldots, z_{q}(t)\right), \quad t \in\right] 0, T[,
$$

where

$z_{i}(t)= \begin{cases}y\left(b_{i}, t\right), \quad b_{i} \in \bar{\Omega} & \begin{array}{l}\text { in the pointwise } \\ \text { case, }\end{array} \\ \int_{D_{i}} y(x, t) f(x) \mathrm{d} x, D_{i} \subset \bar{\Omega} & \text { in the zonal case. }\end{cases}$

Definition 2. A sequence of sensors $\left(D_{i}, f_{i}\right)_{1 \leq i \leq q}$ is said to be $\Gamma$-strategic if the observed system is weakly observable on $\Gamma$, which is equivalent to $\operatorname{Ker}\left(K \gamma_{0}^{*} \chi_{\Gamma}^{*}\right)=\{0\}$.

We define the restriction operators

$$
\chi_{\Gamma}^{1}: H^{\frac{1}{2}}(\partial \Omega) \longrightarrow H^{\frac{1}{2}}(\Gamma)
$$

and

$$
\chi_{\Gamma}^{2}: H^{\frac{3}{2}}(\partial \Omega) \longrightarrow H^{\frac{3}{2}}(\Gamma),
$$

while $\chi_{\Gamma}^{1 *}$ and $\chi_{\Gamma}^{2 *}$ denote respectively the adjoints of $\chi_{\Gamma}^{1}$ and $\chi_{\Gamma}^{2}$. Let the trace function be given by

$$
\gamma_{0}^{1}: H^{1}(\Omega) \longrightarrow H^{\frac{1}{2}}(\partial \Omega)
$$

and

$$
\gamma_{0}^{2}: H^{2}(\Omega) \longrightarrow H^{\frac{3}{2}}(\partial \Omega),
$$

where $\gamma_{0}^{1 *}$ and $\gamma_{0}^{2 *}$ denote respectively the adjoints of $\gamma_{0}^{1}$ and $\gamma_{0}^{2}$.

Assume that $\left(\chi_{\Gamma}^{1} \gamma_{0}^{1} w_{m_{j}}\right)_{1 \leq j \leq r_{m} ; 1 \leq m}$ form a complete set in $H^{\frac{1}{2}}(\Gamma)$ and that $\left(\chi_{\Gamma}^{2} \gamma_{0}^{2} w_{m_{j}}\right)_{1 \leq j \leq r_{m} ; 1 \leq m}$ form a complete set in $H^{\frac{3}{2}}(\Gamma)$. Suppose that $r=\sup r_{m}<\infty$. Then we have the following result.

Proposition 3. If the observation time $T$ is large enough, the sequence of sensors $\left(D_{i}, f_{i}\right)_{1 \leq i \leq q}$ is $\Gamma$-strategic if and only if

(i) $q \geq r$,

(ii) $\operatorname{rank} G_{m}=r_{m}, \forall m \geq 1$,

where

$$
\left(G_{m}\right)_{i, j}
$$

$$
= \begin{cases}\left\langle w_{m_{j}}, f_{i}\right\rangle_{L^{2}\left(\Omega_{i}\right)} & \text { for the zonal case, } \\ w_{m_{j}}\left(b_{i}\right) & \text { for the pointwise case, }\end{cases}
$$

for $1 \leq i \leq q$ and $1 \leq j \leq r_{m}$. 
Proof. (Sufficiency) Let us show that if rank $G_{m}=$ $r_{m}, \forall m \geq 1$, then the system (1)- (9) is weakly observable on $\Gamma$.

We suppose that $\operatorname{Ker}\left(K \gamma_{0}^{*} \chi_{\Gamma}^{*}\right) \neq\{0\}$. Then there exists $z^{*}=\left(z_{1}^{*}, z_{2}^{*}\right) \in H^{\frac{3}{2}}(\Gamma) \times H^{\frac{1}{2}}(\Gamma)$ such that $\left(z_{1}^{*}, z_{2}^{*}\right) \neq 0$ and $K \gamma_{0}^{*} \chi_{\Gamma}^{*} z^{*}=0$. Therefore

$$
\begin{aligned}
& K \gamma_{0}^{*} \chi_{\Gamma}^{*} z^{*} \\
& =\sum_{m=1}^{\infty} \sum_{j=1}^{r_{m}}\left[\left\langle\gamma_{0}^{2 *} \chi_{\Gamma}^{2 *} z_{1}^{*}, w_{m j}\right\rangle \cos \sqrt{-\lambda_{m}} t\right. \\
& \left.\quad+\frac{1}{\sqrt{-\lambda_{m}}}\left\langle\gamma_{0}^{1 *} \chi_{\Gamma}^{1 *} z_{2}^{*}, w_{m j}\right\rangle \sin \sqrt{-\lambda_{m}} t\right] \\
& \quad \times\left\langle w_{m j}, f_{i}\right\rangle=0, \quad \forall i=1, q,
\end{aligned}
$$

where $\psi_{m_{j}}^{1}=\chi_{\Gamma}^{1} \gamma_{0}^{1} w_{m_{j}}$ and $\psi_{m_{j}}^{2}=\chi_{\Gamma}^{2} \gamma_{0}^{2} w_{m_{j}}$.

For $T$ large enough, the set

$$
\left\{\cos \left(\sqrt{-\lambda_{m}} \cdot\right), \sin \left(\sqrt{-\lambda_{m}} \cdot\right)\right\}_{m \geq 1}
$$

forms a complete set in $L^{2}(0, T)$, which gives

$$
\left\{\begin{array}{c}
\sum_{j=1}^{r_{m}}\left\langle\gamma_{0}^{2 *} \chi_{\Gamma}^{2 *} z_{1}^{*}, w_{m j}\right\rangle\left\langle w_{m j}, f_{i}\right\rangle=0, \\
\forall m \geq 1, \quad \forall i=1, \ldots, q . \\
\sum_{j=1}^{r_{m}}\left\langle\gamma_{0}^{1 *} \chi_{\Gamma}^{1 *} z_{2}^{*}, w_{m j}\right\rangle\left\langle w_{m j}, f_{i}\right\rangle=0, \\
\forall m \geq 1, \quad \forall i=1, \ldots, q
\end{array}\right.
$$

i.e.,

$$
\left\{\begin{array}{c}
\sum_{j=1}^{r_{m}}\left\langle z_{1}^{*}, \chi_{\Gamma}^{2} \gamma_{0}^{2} w_{m j}\right\rangle\left\langle w_{m j}, f_{i}\right\rangle=0, \\
\forall m \geq 1, \quad \forall i=1, \ldots, q . \\
\sum_{j=1}^{r_{m}}\left\langle z_{2}^{*}, \chi_{\Gamma}^{1} \gamma_{0}^{1} w_{m j}\right\rangle\left\langle w_{m j}, f_{i}\right\rangle=0 \\
\forall m \geq 1, \quad \forall i=1, \ldots, q .
\end{array}\right.
$$

Since $z_{2}^{*} \in H^{\frac{1}{2}}(\Gamma)$, we obtain

$$
z_{2}^{*}=\sum_{m=1}^{\infty} \sum_{j=1}^{r_{m}}\left\langle z_{2}^{*}, \chi_{\Gamma}^{1} \gamma_{0}^{1} w_{m j}\right\rangle_{H^{\frac{1}{2}}(\Gamma)} \chi_{\Gamma}^{1} \gamma_{0}^{1} w_{m j}
$$

If $z_{2}^{*} \neq 0$, there exists $m_{0} \geq 1$ and $1 \leq j_{0} \leq r_{m_{0}}$ such that

$$
\left\langle z_{2}^{*}, \chi_{\Gamma}^{1} \gamma_{0}^{1} w_{m_{0 j_{0}}}\right\rangle_{H^{\frac{1}{2}(\Gamma)}} \neq 0 .
$$

Consider

$$
\begin{aligned}
z_{m_{0}}^{2}=\left[\left\langle z_{2}^{*}, \chi_{\Gamma}^{1} \gamma_{0}^{1} w_{m_{01} 1}\right\rangle_{H^{\frac{1}{2}}(\Gamma)}, \ldots,\right. \\
\left.\left\langle z_{2}^{*}, \chi_{\Gamma}^{1} \gamma_{0}^{1} w_{m_{0} r_{m_{0}}}\right\rangle_{H^{\frac{1}{2}}(\Gamma)}\right]^{T} .
\end{aligned}
$$

Using (10), we obtain

$$
G_{m_{0}} z_{m_{0}}^{2}=0,
$$

which shows that rank $G_{m_{0}} \neq r_{m_{0}}$. Similar results can be obtained if we take $z_{1}^{*} \neq 0$.

(Necessity) Conversely, we show that if the system (1)-9) is weakly observable on $\Gamma$, then

$$
\operatorname{rank} G_{m}=r_{m}, \quad \forall m \geq 1 .
$$

Suppose that there exists $m_{0} \geq 1$ such that rank $G_{m_{0}} \neq$ $r_{m_{0}}$, that is, there exists

$$
z_{m_{0}}=\left(z_{m_{01}}, \ldots, z_{m_{0} r_{m_{0}}}\right)^{T} \neq 0
$$

and

$$
G_{m_{0}} z_{m_{0}}=0 .
$$

Let $z_{2}^{*} \in H^{\frac{1}{2}}(\Gamma)$ and $z_{1}^{*} \in H^{\frac{3}{2}}(\Gamma)$ satisfying

$$
\begin{cases}\left\langle z_{2}^{*}, \chi_{\Gamma}^{1} \gamma_{0}^{1} w_{m_{0} j}\right\rangle=z_{m_{0} j}, & \forall j=1, \ldots, r_{m_{0}}, \\ \left\langle z_{2}^{*}, \chi_{\Gamma}^{1} \gamma_{0}^{1} w_{m j}\right\rangle=0, & \forall m \neq m_{0} \text { and } \\ & \forall j=1, \ldots, r_{m},\end{cases}
$$

and

$$
\begin{cases}\left\langle z_{1}^{*}, \chi_{\Gamma}^{2} \gamma_{0}^{2} w_{m_{0} j}\right\rangle=z_{m_{0} j}, & \forall j=1, \ldots, r_{m_{0}}, \\ \left\langle z_{1}^{*}, \chi_{\Gamma}^{2} \gamma_{0}^{2} w_{m j}\right\rangle=0, & \forall m \neq m_{0} \text { and } \\ & \forall j=1, \ldots, r_{m} .\end{cases}
$$

Since $G_{m_{0}} z_{m_{0}}=0$ and using (10)-12), the system is not weakly observable on $\Gamma$ which contradicts the above assumption.

Remark 2. The choice of $q=1$ can be sufficient to ensure the system observability. Indeed, we can show that by means of a weak perturbation of the boundary of the system domain $\Omega$, the multiplicity of the eigenvalues may be reduced to one.

In the following, we shall give two approaches which enable the reconstruction of the initial conditions on the boundary part $\Gamma$ of $\partial \Omega$.

\section{Boundary state reconstruction}

4.1. Direct approach. Let us consider the decomposition of the initial state $\bar{y}^{0}$ in the form

$$
\bar{y}^{0}= \begin{cases}\bar{y}_{1}^{0} & \text { on } \Gamma, \\ \bar{y}_{2}^{0} & \text { on } \partial \Omega \backslash \Gamma, \\ \bar{y}_{3}^{0} & \text { in } \Omega,\end{cases}
$$

with $\bar{y}_{1}^{0}=\left(y_{1}^{0}, y_{1}^{1}\right), \bar{y}_{2}^{0}=\left(y_{2}^{0}, y_{2}^{1}\right), \bar{y}_{3}^{0}=\left(y_{3}^{0}, y_{3}^{1}\right)$. Our objective is the reconstruction of $\bar{y}^{0}$, the initial state on $\Gamma$. 
Let $\varepsilon$ be the observation error between the output function $\bar{z}$ and the observation model given by

$$
\varepsilon\left(\bar{y}_{1}^{0}, \bar{y}_{2}^{0}, \bar{y}_{3}^{0}\right)=\left\|\bar{z}-K \bar{y}^{0}\right\|_{\mathcal{O}}^{2}
$$

where $\varepsilon$ is to be meant as a function of the initial state and its components.

Consider the following optimization problem:

$$
\left\{\begin{array}{l}
\min \varepsilon\left(\bar{y}_{1}^{0}, \bar{y}_{2}^{0}, \bar{y}_{3}^{0}\right) \\
\bar{y}_{1}^{0} \in H^{\frac{3}{2}}(\Gamma) \times H^{\frac{1}{2}}(\Gamma) .
\end{array}\right.
$$

Theorem 1. If the system (1) is observable in $\bar{\Omega}$, then the problem (13) admits a unique solution given by $\bar{y}_{1}^{0}=D^{\dagger} \Psi$, which coincides with the regional initial state to be observed on the boundary part $\Gamma$, where $D^{\dagger}$ denotes the pseudoinverse of $D, D$ and $\Psi$ are given by

$$
\left\{\begin{aligned}
D= & B \gamma_{0}^{*} \chi_{\Gamma}^{*}\left(\chi_{\Gamma} \gamma_{0} B^{*} B \gamma_{0}^{*} \chi_{\Gamma}^{*}\right)^{-1} \chi_{\Gamma} \gamma_{0} B^{*} B \\
\Psi= & \left(I-B\left(B^{*} B\right)^{-1}+B \gamma_{0}^{*} \chi_{\Gamma}^{*}\left(\chi_{\Gamma} \gamma_{0} B^{*} B \gamma_{0}^{*} \chi_{\Gamma}^{*}\right)^{-1}\right. \\
& \times \chi_{\Gamma} \gamma_{0} B^{*} \nu \\
R= & \gamma_{0} K^{*} \\
M= & \left(R R^{*}\right)^{-1} \\
\nu= & \left(I+R^{*} M R-K\left(K^{*} K\right)^{-1} K^{*}\right) \\
B= & R^{*} M R K
\end{aligned}\right.
$$

Proof. We have

$$
R R^{*} \in \mathcal{L}\left(H^{\frac{3}{2}}(\partial \Omega) \times H^{\frac{1}{2}}(\partial \Omega), H^{\frac{3}{2}}(\partial \Omega) \times H^{\frac{1}{2}}(\partial \Omega)\right)
$$

and

$$
\begin{aligned}
\left\langle R R^{*} z^{*}, z^{*}\right\rangle=\left\|K \gamma_{0}^{*} z^{*}\right\|^{2} & \leq c\left\|z^{*}\right\|_{H^{\frac{3}{2}}}^{2}(\partial \Omega) \times H^{\frac{1}{2}}(\partial \Omega) \\
\forall z^{*} & \in H^{\frac{3}{2}}(\partial \Omega) \times H^{\frac{1}{2}}(\partial \Omega),
\end{aligned}
$$

which shows that the operator $R R^{*}$ is invertible.

Solution of the problem (13) can be implemented using the followings steps:

Step 1: We minimize $\varepsilon\left(\bar{y}_{1}^{0}, \bar{y}_{2}^{0}, \bar{y}_{3}^{0}\right)$ with respect to $\bar{y}_{3}^{0}$. By developing $\varepsilon$ and using the fact that $\bar{y}^{0}=\bar{y}_{1}^{0 *}+\bar{y}_{2}^{0 *}+\bar{y}_{3}^{0 *}$, where

$$
\begin{aligned}
& \bar{y}_{1}^{0 *}=\left\{\begin{array}{lll}
\bar{y}_{1}^{0} & \text { in } & \Gamma, \\
0 & \text { on } & \bar{\Omega} \backslash \Gamma,
\end{array}\right. \\
& \bar{y}_{2}^{0 *}=\left\{\begin{array}{lll}
\bar{y}_{2}^{0} & \text { in } & \partial \Omega \backslash \Gamma, \\
0 & \text { on } & \bar{\Omega} \cup \Gamma,
\end{array}\right. \\
& \bar{y}_{3}^{0 *}=\left\{\begin{array}{lll}
\bar{y}_{3}^{0} & \text { in } & \Omega, \\
0 & \text { on } & \partial \Omega,
\end{array}\right.
\end{aligned}
$$

we obtain the following problem:

$$
\left\{\begin{array}{l}
\min \Psi_{1}\left(\bar{y}_{3}^{0 *}\right) \\
\gamma_{0} K^{*}\left(K \bar{y}_{3}^{0 *}\right)=0
\end{array}\right.
$$

where

$$
\begin{aligned}
\Psi_{1}\left(\bar{y}_{3}^{0 *}\right)=\left\langle K \bar{y}_{3}^{0 *}\right. & \left., K \bar{y}_{3}^{0 *}\right\rangle_{\mathcal{O}} \\
& +2\left\langle K \bar{y}_{3}^{0 *}, K\left(\bar{y}_{1}^{0 *}+\bar{y}_{2}^{0 *}\right)-\bar{z}\right\rangle_{\mathcal{O}}
\end{aligned}
$$

Consider the Lagrangian operator defined by

$$
\begin{aligned}
\Lambda\left(\bar{y}_{3}^{0 *},\right. & \left.\lambda_{1}\right) \\
= & \left\langle K \bar{y}_{3}^{0 *}, K \bar{y}_{3}^{0 *}\right\rangle_{\mathcal{O}}+2\left\langle K \bar{y}_{3}^{0 *}, K\left(\bar{y}_{1}^{0 *}+\bar{y}_{2}^{0 *}\right)-\bar{z}\right\rangle_{\mathcal{O}} \\
& +\left\langle\lambda_{1}, \gamma_{0} K^{*} K \bar{y}_{3}^{0 *}\right\rangle_{H^{\frac{3}{2}}(\partial \Omega) \times H^{\frac{1}{2}}(\partial \Omega)}
\end{aligned}
$$

Then the condition

$$
\frac{\partial \Lambda\left(\bar{y}_{3}^{0 *}, \lambda_{1}\right)}{\partial \bar{y}_{3}^{0 *}}=0
$$

is equivalent to

$$
\begin{aligned}
2 K^{*} K \bar{y}_{3}^{0 *}+2 K^{*} K\left(\bar{y}_{1}^{0 *}\right. & \left.+\bar{y}_{2}^{0 *}\right) \\
& -2 K^{*} \bar{z}+K^{*} K \gamma_{0}^{*} \lambda_{1}=0
\end{aligned}
$$

Since the system (1) is observable in $\Omega,\left(K^{*} K\right)^{-1}$ exists (Amouroux et al., 1994), which allows us to write

$$
\bar{y}_{3}^{0 *}=-\left(\bar{y}_{1}^{0 *}+\bar{y}_{2}^{0 *}\right)+\left(K^{*} K\right)^{-1} K^{*} \bar{z}-\frac{1}{2} \gamma_{0}^{*} \lambda_{1} .
$$

The constraint $\gamma_{0} K^{*} K \bar{y}_{3}^{0 *}=0$ from the condition $\bar{y}_{3}^{0 *}=$ 0 on $\partial \Omega$ gives

$-\gamma_{0} K^{*} K\left(\bar{y}_{1}^{0 *}+\bar{y}_{2}^{0 *}\right)+\gamma_{0} K^{*} \bar{z}-\frac{1}{2} \gamma_{0}^{*}\left(K^{*} K\right) \gamma_{0}^{*} \lambda_{1}=0$,

and then

$$
\lambda_{1}=2 M R\left(\bar{z}-K\left(\bar{y}_{1}^{0 *}+\bar{y}_{2}^{0 *}\right)\right),
$$

where $M$ and $R$ are given by (14).

Consequently,

$$
\begin{aligned}
\tilde{y}_{3}^{0 *}= & \left(\gamma_{0}^{*} M R K-I\right)\left(\bar{y}_{1}^{0 *}+\bar{y}_{2}^{0 *}\right) \\
& +\left(\left(K^{*} K\right)^{-1} K^{*}-\gamma_{0}^{*} M R\right) \bar{z} .
\end{aligned}
$$

Then the minimum is given by the following equivalent problem:

$$
\varepsilon\left(\bar{y}_{1}^{0 *}, \bar{y}_{2}^{0 *}, \tilde{y}_{3}^{0 *}\right)=\left\|\nu \bar{z}-B\left(\bar{y}_{1}^{0 *}+\bar{y}_{2}^{0 *}\right)\right\|_{\mathcal{O}}^{2},
$$

where $B$ and $\nu$ are given by (14).

Step 2: We minimize $\varepsilon\left(\bar{y}_{1}^{0 *}, \bar{y}_{2}^{0 *}, \tilde{y}_{3}^{0 *}\right)$ with respect to $\bar{y}_{2}^{0 *}$, as in the first step, by developing (16). We obtain the following problem:

$$
\left\{\begin{array}{l}
\min \Psi_{2}\left(\bar{y}_{2}^{0 *}\right) \\
\chi_{\Gamma} \gamma_{0} B^{*} B\left(\bar{y}_{2}^{0 *}\right)=0
\end{array}\right.
$$

where

$$
\Psi_{2}\left(\bar{y}_{2}^{0 *}\right)=\left\langle B \bar{y}_{2}^{0 *}, B \bar{y}_{2}^{0 *}\right\rangle_{\mathcal{O}}+2\left\langle B \bar{y}_{2}^{0 *}, B \bar{y}_{2}^{0 *}-\nu \bar{z}\right\rangle_{\mathcal{O}} .
$$


The Lagrangian is given by

$$
\begin{aligned}
\Lambda_{2}\left(\bar{y}_{2}^{0 *}, \lambda_{2}\right)= & \left\langle B \bar{y}_{2}^{0 *}, B \bar{y}_{2}^{0 *}\right\rangle_{\mathcal{O}}+2\left\langle B \bar{y}_{2}^{0 *}, B \bar{y}_{2}^{0 *}-\nu \bar{z}\right\rangle_{\mathcal{O}} \\
& +\left\langle\lambda_{2}, \chi_{\Gamma} \gamma_{0} B^{*} B \bar{y}_{2}^{0 *}\right\rangle_{H^{\frac{3}{2}}}(\Gamma) \times H^{\frac{1}{2}}(\Gamma)
\end{aligned}
$$

Then the condition

$$
\frac{\partial \Lambda\left(\bar{y}_{2}^{0 *}, \lambda_{2}\right)}{\partial \bar{y}_{2}^{0 *}}=0
$$

is equivalent to

$$
2 B^{*} B \bar{y}_{2}^{0 *}+2 B^{*} B \bar{y}_{1}^{0 *}-2 B^{*} \nu \bar{z}+B^{*} B \gamma_{0}^{*} \chi_{\Gamma}^{*} \lambda_{2}=0 .
$$

We obtain

$$
\bar{y}_{2}^{0 *}=-\bar{y}_{1}^{0 *}+\left(B^{*} B\right)^{-1} B^{*} \nu \bar{z}-\frac{1}{2} \gamma_{0}^{*} \chi_{\Gamma}^{*} \lambda_{2} .
$$

The constraint $\chi_{\Gamma} \gamma_{0} B^{*} B\left(\bar{y}_{2}^{0 *}\right)=0$, which comes from $\bar{y}_{2}^{0 *}=0$ on $\Omega \cup \Gamma$, gives

$-\chi_{\Gamma} \gamma_{0} B^{*} B \bar{y}_{1}^{0 *}+\chi_{\Gamma} \gamma_{0} B^{*} \nu \bar{z}-\frac{1}{2} \chi_{\Gamma} \gamma_{0} B^{*} B \gamma_{0}^{*} \chi_{\Gamma}^{*} \lambda_{2}=0$.

We obtain

$$
\begin{aligned}
\lambda_{2}=2 & \left(\chi_{\Gamma} \gamma_{0} B^{*} B \gamma_{0}^{*} \chi_{\Gamma}^{*}\right)^{-1} \chi_{\Gamma} \gamma_{0} B^{*} \nu \bar{z} \\
& -2\left(\chi_{\Gamma} \gamma_{0} B^{*} B \gamma_{0}^{*} \chi_{\Gamma}^{*}\right)^{-1} \chi_{\Gamma} \gamma_{0} B^{*} B \bar{y}_{1}^{0 *} .
\end{aligned}
$$

Thus

$$
\begin{aligned}
\tilde{y}_{2}^{0 *}= & -\bar{y}_{1}^{0 *}+\left[\left(B^{*} B\right)^{-1}\right. \\
& \left.-\gamma_{0}^{*} \chi_{\Gamma}^{*}\left(\chi_{\Gamma} \gamma_{0} B^{*} B \gamma_{0}^{*} \chi_{\Gamma}^{*}\right)^{-1} \chi_{\Gamma} \gamma_{0}\right] B^{*} \nu \bar{z} \\
& +\gamma_{0}^{*} \chi_{\Gamma}^{*}\left(\chi_{\Gamma} \gamma_{0} B^{*} B \gamma_{0}^{*} \chi_{\Gamma}^{*}\right)^{-1} \chi_{\Gamma} \gamma_{0} B^{*} B \bar{y}_{1}^{0 *} .
\end{aligned}
$$

The minimum is then given by the equivalent problem

$$
\varepsilon\left(\bar{y}_{1}^{0 *}, \tilde{y}_{2}^{0 *}, \tilde{y}_{3}^{0 *}\right)=\left\|\Psi \bar{z}-D \bar{y}_{1}^{0 *}\right\|_{\mathcal{O}}^{2},
$$

where $\Psi$ and $D$ are given by (14).

Step 3: The solution of the problem (13) turns out to solve the problem $\varepsilon\left(\bar{y}_{1}^{0 *}, \tilde{y}_{2}^{0 *}, \tilde{y}_{3}^{0 *}\right)$ given by (18) with respect to $\bar{y}_{1}^{0 *}$. We have

$$
\begin{aligned}
& \varepsilon\left(\bar{y}_{1}^{0 *}, \tilde{y}_{2}^{0 *}, \tilde{y}_{3}^{0 *}\right) \\
& =\left\langle D \bar{y}_{1}^{0 *}, D \bar{y}_{1}^{0 *}\right\rangle_{\mathcal{O}}-2\left\langle D \bar{y}_{1}^{0 *}, \Psi \bar{z}\right\rangle_{\mathcal{O}}+\langle\Psi \bar{z}, \Psi \bar{z}\rangle_{\mathcal{O}} .
\end{aligned}
$$

The condition

$$
\frac{\varepsilon\left(\bar{y}_{1}^{0 *}, \tilde{y}_{2}^{0 *}, \tilde{y}_{3}^{0 *}\right)}{\partial \bar{y}_{1}^{0 *}}=0
$$

gives

$$
2 D^{*} D \bar{y}_{1}^{0 *}-2 D^{*} \Psi \bar{z}=0 .
$$

Thus

$$
\tilde{y}_{1}^{0 *}=\left(D^{*} D\right)^{-1} D \Psi \bar{z}=D^{\dagger} \Psi \bar{z} .
$$

Since the operator $(18)$ is strictly convex, then $\tilde{y}_{1}^{0 *}$ is unique.
4.2. Hilbert uniqueness method approach. The subject of this section is to update the Hilbert uniqueness method developed by Lions (1988) to the case of regional observability of following hyperbolic system:

$$
\left\{\begin{aligned}
\frac{\partial^{2} y(x, t)}{\partial t^{2}} & =\Delta y(x, t), & & Q, \\
y(x, 0) & =y^{0}(x), & \frac{\partial y}{\partial t}(x, 0)=y^{1}(x), & \Omega, \\
\frac{\partial y(\zeta, t)}{\partial \eta} & =0, & & \Sigma,
\end{aligned}\right.
$$

where $\Delta$ is the Laplacian operator and we assume that (19) is augmented with the output function

$$
z(t)=\langle y(t), f\rangle_{L^{2}(D)},
$$

where $f \in L^{2}(D)$.

We consider the following decomposition:

$$
\begin{aligned}
y^{0} & = \begin{cases}y_{1}^{0} & \text { in } \omega, \\
y_{2}^{0} & \text { in } \Omega \backslash \omega,\end{cases} \\
y^{1} & =\left\{\begin{array}{lll}
y_{1}^{1} & \text { in } \omega, \\
y_{2}^{1} & \text { in } \Omega \backslash \omega .
\end{array}\right.
\end{aligned}
$$

In the sequel, without loss of generality, we assume that the eigenfunctions $\left(w_{m}\right)_{m \geq 1}$ of the operator $\Delta$ associated with the eigenvalues are simple.

Consider the set

$$
\begin{gathered}
G=\left\{\chi_{\omega}^{*} \chi_{\omega}\left(\varphi^{0}, \varphi^{1}\right) \mid\left(\varphi^{0}, \varphi^{1}\right) \in \mathcal{D}(\mathcal{A}) \times H^{1}(\Omega)\right. \\
\text { with } \left.\varphi^{0}=\varphi^{1}=0 \text { in } \Omega \backslash \omega\right\} .
\end{gathered}
$$

For $\left(\varphi^{0}, \varphi^{1}\right) \in G$, the system

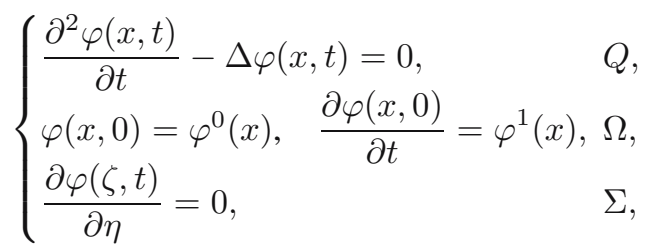

admits a unique solution

$\varphi \in \mathcal{C}\left(0, T ; H^{2}(\Omega)\right) \cap \mathcal{C}^{1}\left(0, T ; H^{1}(\Omega)\right) \cap \mathcal{C}^{2}\left(0, T ; L^{2}(\Omega)\right)$

(see (Lions and Magenes, 1968)).

We define a semi-norm on $G$ by

$$
\left\|\left(\varphi^{0}, \varphi^{1}\right)\right\|_{G}=\left[\int_{0}^{T}\langle\varphi(t), f\rangle_{L^{2}(D)}^{2} \mathrm{~d} t\right]^{\frac{1}{2}},
$$

and we consider the retrograde system

$$
\left\{\begin{array}{rlrl}
\frac{\partial^{2} \psi(x, t)}{\partial t} & =\Delta \psi(x, t)+\langle\varphi(t), f\rangle_{L^{2}(D)} \chi_{D} f(x), & & Q, \\
\psi(x, T) & =0, \quad \frac{\partial \psi(x, T)}{\partial t}=0, & \Omega, \\
\frac{\partial \psi(\zeta, t)}{\partial \eta} & =0 & \Sigma,
\end{array}\right.
$$


which admits a unique solution

$\psi \in \mathcal{C}\left(0, T ; H^{2}(\Omega)\right) \cap \mathcal{C}^{1}\left(0, T ; H^{1}(\Omega)\right) \cap \mathcal{C}^{2}\left(0, T ; L^{2}(\Omega)\right)$

(see (Lions and Magenes, 1968)).

Let the operator $\Lambda$ be defined by

$$
\Lambda\left(\varphi^{0}, \varphi^{1}\right)=\mathcal{P}(-\dot{\psi}(0), \psi(0))
$$

where $\mathcal{P}=\chi_{\omega}^{*} \chi_{\omega}$ and $\chi_{\omega}^{*}$ is the adjoint operator of $\chi_{\omega}$.

Consider the system

$$
\left\{\begin{aligned}
\frac{\partial^{2} \bar{z}(x, t)}{\partial t} & =\Delta \bar{z}(x, t)+z(t) \chi_{D}(x) f(x), & & Q, \\
\bar{z}(x, T) & =0, \quad \frac{\partial \bar{z}(x, T)}{\partial t}=0, & & \Omega, \\
\frac{\partial \bar{Z}(\zeta, t)}{\partial \eta} & =0, & & \Sigma .
\end{aligned}\right.
$$

We have $\bar{z}(0)=\bar{z}^{0}, \bar{z}^{\prime}(0)=\bar{z}^{1}, \psi(0)=\psi^{0}$ and $\psi^{\prime}(0)=$ $\psi^{1}$.

If $\varphi^{0}, \varphi^{1}$ are convenably chosen on $G$, i.e., such that $\bar{z}^{0}=\psi^{0}$ and $\bar{z}^{1}=\psi^{1}$ in $\omega$, then the observability problem for the system (19) in the subregion $\omega$ amounts to solving the equation

$$
\Lambda\left(\varphi^{0}, \varphi^{1}\right)=\mathcal{P}\left(-\bar{z}^{1}, \bar{z}^{0}\right) .
$$

Theorem 2. If the system (19) augmented with the output function (20) is weakly observable in $\omega$, then the equation (25) admits a unique solution $\left(\varphi^{0}, \varphi^{1}\right) \in G$, which coincides with the initial conditions $\left(y_{1}^{0}, y_{1}^{1}\right)$ in the subregion $\omega$, and the initial conditions to be observed in the subregion $\Gamma$ of $\partial \Omega$ are given by

$$
\left(y_{\Gamma}^{0}, y_{\Gamma}^{1}\right)=\chi_{\Gamma} \gamma_{0}\left(\varphi^{0}, \varphi^{1}\right) .
$$

Proof. (Part 1) We show that if the sensor $(D, f)$ is $\omega$ strategic, then the formula (22) defines a seminorm on $G$. Indeed,

$$
\begin{aligned}
\left\|\left(\varphi^{0}, \varphi^{1}\right)\right\|_{G}=0 & \Leftrightarrow\langle\varphi(t), f\rangle_{L^{2}(D)}=0 \\
& \Leftrightarrow \mathcal{C} S(t)\left(\varphi^{0}, \varphi^{1}\right)=0,
\end{aligned}
$$

where $(S(t))_{t \geq 0}$ is the semigroup generated by $\left(\begin{array}{cc}0 & I \\ \Delta & 0\end{array}\right)$. Then we have

$$
K(t) \chi_{\omega}^{*} \chi_{\omega}\left(\tilde{\varphi}^{0}, \tilde{\varphi}^{1}\right)=0
$$

with

$$
\left(\varphi^{0}, \varphi^{1}\right)=\chi_{\omega}^{*} \chi_{\omega}\left(\tilde{\varphi}^{0}, \tilde{\varphi}^{1}\right) .
$$

Since the system (1) is weakly $\omega$-observable, we have

$$
\chi_{\omega}\left(\tilde{\varphi}^{0}, \tilde{\varphi}^{1}\right)=0
$$

and then

$$
\left(\tilde{\varphi}^{0}, \tilde{\varphi}^{1}\right)=0
$$

Consequently, $\varphi^{0}=\varphi^{1}=0$. Thus 22 is a norm.

(Part 2) Let $\hat{G}$ be the completion set of $G$ with respect to the norm (22) equipped with the associated inner product $\langle\cdot, \cdot\rangle_{\hat{G}}$ and $\hat{G}^{*}$ be its dual. We show that $\Lambda$ is an isomorphism from $\hat{G}$ onto $\hat{G}^{*}$.

Indeed,

$$
\left\langle\Lambda\left(\varphi^{0}, \varphi^{1}\right),\left(\varphi^{0}, \varphi^{1}\right)\right\rangle=\left\langle\left(-\psi^{1}, \psi^{0}\right),\left(\varphi^{0}, \varphi^{1}\right)\right\rangle .
$$

On the other hand, multiplying (23) by $\varphi$ (the solution to (21) ) and integrating the result by part, we obtain

$$
\begin{aligned}
& \int_{0}^{T}\left\langle\psi^{\prime \prime}(t), \varphi(t)\right\rangle \mathrm{d} t \\
&=\left\langle\psi^{\prime}(T), \varphi(T)\right\rangle-\left\langle\psi^{\prime}(0), \varphi^{0}\right\rangle \\
&-\left\langle\psi(T), \varphi^{\prime}(T)\right\rangle+\left\langle\psi(0), \varphi^{1}\right\rangle \\
&+\int_{0}^{T}\left\langle\psi(t), \varphi^{\prime \prime}(t)\right\rangle \mathrm{d} t \\
&= \int_{0}^{T}\left\langle\psi(t), \varphi^{\prime \prime}(t)\right\rangle \mathrm{d} t \\
&+\left\langle\left(-\psi^{\prime}(0), \psi(0)\right),\left(\varphi^{0}, \varphi^{1}\right)\right\rangle
\end{aligned}
$$

Using Green formulae, we have

$$
\begin{aligned}
& \int_{0}^{T}\langle\Delta \psi(t), \varphi(t)\rangle \mathrm{d} t \\
& =\int_{0}^{T}\langle\psi(t), \Delta \varphi(t)\rangle \mathrm{d} t+\int_{\sum} \frac{\partial \psi}{\partial \eta} \varphi \mathrm{d} \sigma \\
& \quad-\int_{\sum} \psi \frac{\partial \varphi}{\partial \eta} \mathrm{d} \sigma \\
& =\int_{0}^{T}\langle\psi(t), \Delta \varphi(t)\rangle \mathrm{d} t .
\end{aligned}
$$

Thus

$$
\begin{aligned}
\int_{0}^{T}\left[\left\langle\psi^{\prime \prime}(t), \varphi(t)\right\rangle\right. & -\langle\Delta \psi(t), \varphi(t)\rangle] \mathrm{d} t \\
& =\left\langle\left(-\psi^{\prime}(0), \psi(0)\right),\left(\varphi^{0}, \varphi^{1}\right)\right\rangle .
\end{aligned}
$$

On the other hand, we have

$$
\begin{aligned}
& \int_{0}^{T}\langle\varphi(t), f\rangle_{L^{2}(D)}\left\langle\chi_{D} f, \varphi(t)\right\rangle \mathrm{d} t \\
& \quad=\int_{0}^{T}\langle\varphi(t), f\rangle_{L^{2}(D)}^{2} \mathrm{~d} t=\left\|\left(\varphi^{0}, \varphi^{1}\right)\right\|^{2} .
\end{aligned}
$$

Thus

$$
\left\langle\Lambda\left(\varphi^{0}, \varphi^{1}\right),\left(\varphi^{0}, \varphi^{1}\right)\right\rangle=\left\|\left(\varphi^{0}, \varphi^{1}\right)\right\|^{2} .
$$

Let us consider $\left(\hat{\varphi}^{0}, \hat{\varphi}^{1}\right) \in G$ and $\hat{\varphi}(t)$ the associated solution to 21 . 
Integrating by part and using Green formulae, we obtain

$$
\begin{aligned}
& \left\langle\Lambda\left(\varphi^{0}, \varphi^{1}\right),\left(\hat{\varphi}^{0}, \hat{\varphi}^{1}\right)\right\rangle \\
& \quad=\int_{0}^{T}\langle\varphi(t), f\rangle_{L^{2}(D)}\langle\hat{\varphi}(t), f\rangle_{L^{2}(D)} \mathrm{d} t
\end{aligned}
$$

which gives

$$
\begin{aligned}
&\left|\left\langle\Lambda\left(\varphi^{0}, \varphi^{1}\right),\left(\hat{\varphi}^{0}, \hat{\varphi}^{1}\right)\right\rangle\right| \\
& \leq {\left[\int_{0}^{T}\langle\varphi(t), f\rangle_{L^{2}(D)}^{2} \mathrm{~d} t\right]^{1 / 2} } \\
& {\left[\int\langle\hat{\varphi}(t), f\rangle_{L^{2}(D)}^{2} \mathrm{~d} t\right]^{1 / 2} } \\
& \leq\left\|\left(\varphi^{0}, \varphi^{1}\right)\right\|_{\hat{G}}\left\|\left(\hat{\varphi}^{0}, \hat{\varphi}^{1}\right)\right\|_{\hat{G}}, \\
& \forall\left(\varphi^{0}, \varphi^{1}\right),\left(\hat{\varphi}^{0}, \hat{\varphi}^{1}\right) \in G,
\end{aligned}
$$

which ensures a unique extension of $\Lambda$ to a bounded linear operator. From (31) and (32) it follows that $\Lambda$ is an isomorphism from $\hat{G}$ onto $\hat{G}^{*}$.

Remark 3. If the system (19) is observable by one internal pointwise sensor, we show by the same techniques as in the zonal case that the operator $\Lambda$ is an isomorphism.

\section{Numerical approach}

Here we consider the system (19) and assume that the initial conditions $y^{0}$ and $y^{1}$ are sufficiently regular so that (19) has a regular state (Lions, 1968), and that measurements may be obtained by a pointwise sensor $\left(b, \delta_{b}\right)$ given by

$$
z(t)=y(b, t), \quad t \in] 0, T[.
$$

The objective is to reconstruct the initial conditions $y_{1}^{0}$ and $y_{1}^{1}$ in the subregion $\omega$ from measurements given by (33) along the time interval $] 0, T[$. We apply the trace restriction on a part $\Gamma \subset \partial \Omega \cap \partial \omega$.

\subsection{Approximation formulae.}

Proposition 4. If the sensor $\left(b, \delta_{b}\right)$ is $\omega$-strategic, then, for $T$ large enough, the initial conditions $y_{1}^{0}$ and $y_{1}^{1}$ may be approached by (34) and (35).

Proof. The solution to 25) solves the following minimization problem:

$$
\begin{aligned}
& \Phi\left(\varphi^{0}, \varphi^{1}\right) \\
& =\frac{1}{2}\left\langle\Lambda\left(\varphi^{0}, \varphi^{1}\right),\left(\varphi^{0}, \varphi^{1}\right)\right\rangle-\left\langle\mathcal{P}\left(-\bar{z}^{1}, \bar{z}^{0}\right),\left(\varphi^{0}, \varphi^{1}\right)\right\rangle \\
& =\frac{1}{2} \int_{0}^{T} \varphi^{2}(b, t) \mathrm{d} t-\left\langle-\bar{z}^{1}, \varphi^{0}\right\rangle-\left\langle\bar{z}^{0}, \varphi^{1}\right\rangle .
\end{aligned}
$$

We have

$$
\begin{aligned}
& \int_{0}^{T} \varphi^{2}(b, t) \mathrm{d} t \\
& =\int_{0}^{T}\left[\sum _ { j \geq 1 } \left[\left\langle\varphi^{0}, w_{j}\right\rangle \cos \sqrt{-\lambda_{j}} t+\frac{1}{\sqrt{-\lambda_{j}}}\left\langle\varphi^{1}, w_{j}\right\rangle\right.\right. \\
& \left.\left.\quad \sin \sqrt{-\lambda_{j}} t\right] w_{j}(b)\right]^{2} \mathrm{~d} t .
\end{aligned}
$$

Expanding the integrand and letting $T \longrightarrow+\infty$, we obtain

$$
\begin{aligned}
\lim _{T \longrightarrow+\infty} & \frac{1}{2 T} \int_{0}^{T} \varphi^{2}(b, t) \mathrm{d} t \\
& =\frac{1}{4} \sum_{j \geq 1}\left[\left\langle\varphi^{0}, w_{j}\right\rangle^{2}-\frac{1}{\lambda_{j}}\left\langle\varphi^{1}, w_{j}\right\rangle^{2}\right] w_{j}^{2}(b) .
\end{aligned}
$$

Thus, for $T$ large enough, we obtain

$$
\begin{aligned}
\frac{1}{2} \int_{0}^{T} & \varphi^{2}(b, t) \mathrm{d} t \\
& \simeq \frac{T}{4} \sum_{j \geq 1}\left[\left\langle\varphi^{0}, w_{j}\right\rangle^{2}-\frac{1}{\lambda_{j}}\left\langle\varphi^{1}, w_{j}\right\rangle^{2}\right] w_{j}^{2}(b) .
\end{aligned}
$$

On the other hand,

$$
\varphi^{0}(x)=\sum_{j \geq 1}\left\langle\varphi^{0}, w_{j}\right\rangle_{L^{2}(\omega)} w_{j}(x)
$$

and

$$
\varphi^{1}(x)=\sum_{j \geq 1}\left\langle\varphi^{1}, w_{j}\right\rangle_{L^{2}(\omega)} w_{j}(x)
$$

Then

$$
\left\langle-\bar{z}^{1}, \varphi^{0}\right\rangle_{L^{2}(\omega)}=\sum_{j \geq 1}\left\langle\varphi^{0}, w_{j}\right\rangle_{L^{2}(\omega)}\left\langle-\bar{z}^{1}, w_{j}\right\rangle_{L^{2}(\omega)}
$$

and

$$
\left\langle\bar{z}^{0}, \varphi^{1}\right\rangle_{L^{2}(\omega)}=\sum_{j \geq 1}\left\langle\varphi^{1}, w_{j}\right\rangle_{L^{2}(\omega)}\left\langle\bar{z}^{0}, w_{j}\right\rangle_{L^{2}(\omega)} .
$$

The minimization of 36 is equivalent to finding

$$
\begin{aligned}
& \inf _{\left(\varphi^{0}, \varphi^{1}\right)} \sum_{j \geq 1}\left\{\frac{T}{4}\left[\left\langle\varphi^{0}, w_{j}\right\rangle^{2}-\frac{1}{\lambda_{j}}\left\langle\varphi^{1}, w_{j}\right\rangle^{2}\right] w_{j}^{2}(b)\right. \\
& \left.\quad-\left[\left\langle\varphi^{0}, w_{j}\right\rangle\left\langle-\bar{z}^{1}, w_{j}\right\rangle+\left\langle\varphi^{1}, w_{j}\right\rangle\left\langle\bar{z}^{0}, w_{j}\right\rangle\right]\right\},
\end{aligned}
$$

which is equivalent, by separating variables, to solving the problems

$$
\begin{aligned}
\inf _{\varphi^{0}} \sum_{j \geq 1}\{ & \frac{T}{4}\left\langle\varphi^{0}, w_{j}\right\rangle_{L^{2}(\omega)}^{2} w_{j}^{2}(b) \\
& \left.-\left\langle\varphi^{0}, w_{j}\right\rangle_{L^{2}(\omega)}\left\langle-\bar{z}^{1}, w_{j}\right\rangle_{L^{2}(\omega)}\right\}
\end{aligned}
$$




$$
\begin{aligned}
& \tilde{y}_{1}^{0}= \begin{cases}\frac{2}{T} \sum_{j \geq 1}\left[\frac{1}{w_{j}^{2}(b)} \sum_{k \geq 1} w_{k}(b) \int_{0}^{T} y(b, t) \cos \left(\sqrt{-\lambda_{k}} t\right) \mathrm{d} t\left\langle w_{k}, w_{j}\right\rangle_{L^{2}(\omega)}\right] w_{j}(x), & \forall x \in \omega, \\
0, & \forall x \in \Omega \backslash \omega,\end{cases} \\
& \tilde{y}_{1}^{1}= \begin{cases}\frac{-2}{T} \sum_{j \geq 1}\left[\frac{\lambda_{j}}{w_{j}^{2}(b)} \sum_{k \geq 1} \frac{w_{k}(b)}{\sqrt{-\lambda_{k}}} \int_{0}^{T} y(b, t) \sin \left(\sqrt{-\lambda_{k}} t\right) \mathrm{d} t\left\langle w_{k}, w_{j}\right\rangle_{L^{2}(\omega)}\right] w_{j}(x), & \forall x \in \omega, \\
0, & \forall x \in \Omega \backslash \omega .\end{cases}
\end{aligned}
$$

and

$$
\begin{aligned}
\inf _{\varphi^{1}} \sum_{j \geq 1}\{ & \frac{T}{4 \lambda_{j}}\left\langle\varphi^{1}, w_{j}\right\rangle_{L^{2}(\omega)}^{2} w_{j}^{2}(b) \\
& \left.-\left\langle\varphi^{1}, w_{j}\right\rangle_{L^{2}(\omega)}\left\langle\bar{z}^{0}, w_{j}\right\rangle_{L^{2}(\omega)}\right\}
\end{aligned}
$$

whose solutions are

$$
\left\langle\varphi^{0}, w_{j}\right\rangle_{L^{2}(\omega)}=\frac{2}{T} \frac{\left\langle-\bar{z}^{1}, w_{j}\right\rangle_{L^{2}(\omega)}}{w_{j}^{2}(b)}, \quad \forall j \geq 1
$$

and

$$
\left\langle\varphi^{1}, w_{j}\right\rangle_{L^{2}(\omega)}=\frac{2 \lambda_{j}}{T} \frac{\left\langle-\bar{z}^{0}, w_{j}\right\rangle_{L^{2}(\omega)}}{w_{j}^{2}(b)}, \quad \forall j \geq 1 .
$$

If we set

$$
\bar{z}(x, t)=\sum_{k \geq 1} \bar{z}_{k}(t) w_{k}(x)
$$

with

$$
\bar{z}_{k}(t)=\frac{w_{k}(b)}{\sqrt{-\lambda_{k}}} \int_{0}^{T} z(s) \sin \sqrt{-\lambda_{k}}(s-t) \mathrm{d} s
$$

and

$$
z(s)=y(b, s)
$$

then

$$
\bar{z}(x, 0)=\bar{z}^{0}(x)=\sum_{k \geq 1} \bar{z}_{k}(0) w_{k}(x)
$$

with

$$
\bar{z}_{k}(0)=\frac{w_{k}(b)}{\sqrt{-\lambda_{k}}} \int_{0}^{T} z(s) \sin \sqrt{-\lambda_{k}} s \mathrm{~d} s .
$$

But

$$
\bar{z}^{0}(x)=\sum_{k \geq 1}\left\langle\bar{z}^{0}, w_{k}\right\rangle_{L^{2}(\Omega)} w_{k}(x)
$$

and

$$
\bar{z}^{1}(x)=\sum_{k \geq 1}\left\langle\bar{z}^{1}, w_{k}\right\rangle_{L^{2}(\Omega)} w_{k}(x),
$$

and then

$$
\begin{aligned}
& \left\langle\bar{z}^{0}, w_{k}\right\rangle_{L^{2}(\Omega)} \\
& =\frac{w_{k}(b)}{\sqrt{-\lambda_{k}}} \int_{0}^{T} z(s) \sin \sqrt{-\lambda_{k}} s \mathrm{~d} s, \quad \forall k \geq 1,
\end{aligned}
$$

and

$$
\begin{aligned}
& \left\langle\bar{z}^{1}, w_{k}\right\rangle_{L^{2}(\Omega)} \\
& \quad=-w_{k}(b) \int_{0}^{T} z(s) \cos \sqrt{-\lambda_{k}} s \mathrm{~d} s, \quad \forall k \geq 1 .
\end{aligned}
$$

We obtain

$$
\begin{aligned}
& \left\langle\bar{z}^{0}, w_{j}\right\rangle_{L^{2}(\omega)} \\
& \quad=\sum_{k \geq 1} \frac{w_{k}(b)}{\sqrt{-\lambda_{k}}} \int_{0}^{T} z(s) \sin \sqrt{-\lambda_{k}} s \mathrm{~d} s\left\langle w_{k}, w_{j}\right\rangle_{L^{2}(\omega)}
\end{aligned}
$$

and

$$
\begin{aligned}
& \left\langle\bar{z}^{1}, w_{j}\right\rangle_{L^{2}(\omega)} \\
& \quad=\sum_{k \geq 1} w_{k}(b) \int_{0}^{T} z(s) \cos \sqrt{-\lambda_{k}} s d s\left\langle w_{k}, w_{j}\right\rangle_{L^{2}(\omega)} .
\end{aligned}
$$

Substituting this, we obtain (34) and (35).

5.2. Simulation results. Here we consider the twodimensional system evolving in $\Omega=] 0,1[\times] 0,1[$ :

$$
\begin{cases}\frac{\partial^{2} y\left(x_{1}, x_{2}, t\right)}{\partial t^{2}}-\Delta y\left(x_{1}, x_{2}, t\right)=0, & \Omega \times] 0, T[ \\ y\left(x_{1}, x_{2}, 0\right)=y^{0}\left(x_{1}, x_{2}\right), & \Omega, \\ \frac{\partial y\left(x_{1}, x_{2}, 0\right)}{\partial t}=y^{1}\left(x_{1}, x_{2}\right), & \Omega \\ \frac{\partial y\left(\zeta_{1}, \zeta_{2}, t\right)}{\partial \eta}=0, & \Sigma\end{cases}
$$

Measurements are made by one pointwise sensor:

$$
z(t)=y\left(\left(b_{1}, b_{2}\right), t\right)
$$

where

$$
\left.b_{1}, b_{2} \in \Omega, t \in\right] 0, T[\text {. }
$$




\section{Let}

$$
\begin{cases}y_{\Gamma}^{0}(\zeta)=A \cos (\pi \zeta), & \forall \zeta \in[0,1] \\ y_{\Gamma}^{1}(\zeta)=(1+B) \cos (\pi \zeta), & \forall \zeta \in[0,1]\end{cases}
$$

be the initial conditions to be reconstructed on $\Gamma=\{0\} \times$ $[0,1]$.

$$
\begin{aligned}
& \text { Let } \omega=] 0,0.24[\times] 0,1[\text { and } \\
& \left\{\begin{array}{l}
y^{0}\left(x_{1}, x_{2}\right)=A \cos \left(\pi x_{1}\right) \cos \left(\pi x_{2}\right), \\
y^{1}\left(x_{1}, x_{2}\right)=(1+B) \cos \left(\pi x_{1}\right) \cos \left(\pi x_{2}\right)
\end{array}\right.
\end{aligned}
$$

be the extensions of $y_{\Gamma}^{0}, y_{\Gamma}^{1}$ to $\omega$, where $B_{1}$ and $B_{2}$ are selected to satisfy some numerical requirements (in order to obtain reasonable amplitudes for $y_{1}^{0}$ and $y_{1}^{1}$ ).

Consider the following data:

$$
\begin{gathered}
T=7, \quad A=0.02, \quad B=-0.01, \\
\left(b_{1}, b_{2}\right)=(0.74,0.98) .
\end{gathered}
$$

Applying (34) and (35) truncated to $M=4$, we obtain the results of Figs. 1 and 2.

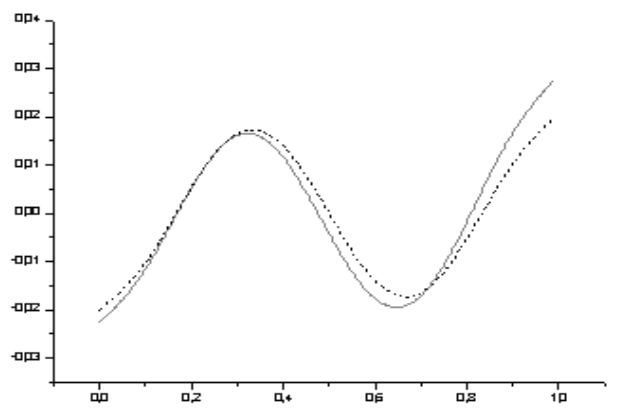

Fig. 1. Initial (dashed line) and estimated (continuous line) state on $\Gamma$.

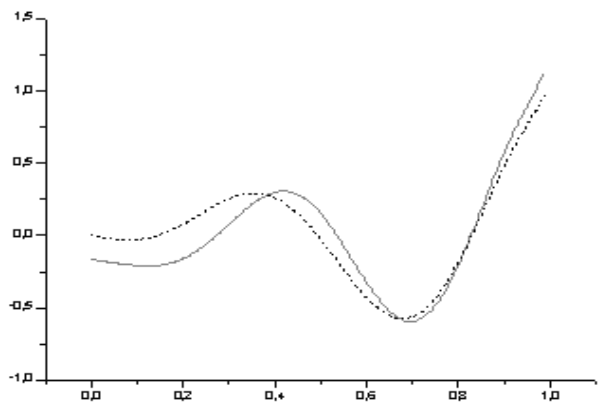

Fig. 2. Initial speed (dashed line) and estimated speed (continuous line) on $\Gamma$.

The reconstruction is obtained with the error

$$
\mathcal{E}^{2}=\left\|y^{0}-y_{e}^{0}\right\|_{L^{2}(\Omega)}^{2}+\left\|y^{1}-y_{e}^{1}\right\|_{L^{2}(\Omega)}^{2}=2.12 \times 10^{-4} \text {. }
$$

\section{Conclusion}

In this paper the question of how to reconstruct the system state and speed on a part of the boundary of the evolution domain has been considered. We explored two approaches: the first using optimization techniques and the second employing a link between the internal and boundary approaches to reconstruct the boundary state on $\Gamma$. The obtained results were verified via a numerical example and simulations. The extension to semilinear systems is of great interest and this problem is currently studied.

\section{References}

Amouroux, A., El Jai, M. and Zerrik, E. (1994). Regional observability of distributed systems, International Journal of Systems Science 25(2): 301-313.

Avdonin, S.A. and Ivanov, S.A. (1995). Families of Exponentials. The Method of Moments in Controllability Problems for Distributed Parameter Systems, Cambridge University Press, New York, NY/London/Melbourne.

Avdonin, S.A. and Ivanov, S.A. (1995). Boundary controllability problems for the wave equation in a parallelepiped, Applied Mathematic Letters 8(2): 97-102.

Avdonin, S.A., Ivanov, S.A. and Joó, I. (1995). Exponential series in the problem of initial and pointwise control of a rectangular vibrating membrane, Studia Scientiarium Mathematicarum Hungarica 30(3-4): 243-259.

Curtain, R.F. and Zwart, H. (1995). An Introduction to Infinite Dimensional Linear Systems Theory, Texts in Applied Mathematics, Vol. 21, Springer-Verlag, New York, NY.

Curtain, R.F. and Pritchard, A.J. (1978). Infinite Dimensional Linear Systems Theory, Springer-Verlag, New York, NY.

Dolecki, S. and Russel, D. (1977). A general theory of observation and control, SIAM Journal of Control 15(2): 185-220.

El Jai, A. and Pritchard, A.J. (1988). Sensors and Actuators in Distributed Systems Analysis, J. Wiley, New York, NY.

Kobayashi, T. (1980). Discrete-time observability for distributed parameter systems, International Journal of Control 31(1): 181-193.

Lions, J.L. and Magenes, E. (1968). Problèmes aux limites non homogènes et applications, Vols. 1 et 2, Dunod, Paris.

Lions, J.L. (1968). Contrôle optimal des systèmes gouvernés par des équations aux dérivées partielles, Dunod, Paris.

Lions, J.L. (1988). Contrôlabilité Exacte. Perturbations et Stabilisation des Systèmes Distribués, Tome 1, Contrôlabilité Exacte, Masson, Paris.

Li, D., Gilliam, Z. and Martin, C. (1988). Discrete observavility of the heat equation on bounded domain, International Journal of Control 48(2): 755-780.

Micheletti, A. M. (1976). Perturbazione dello specttro di Un operatore ellitico di tipo variazionale, in relazione ad Una variazione del compo, Ricerche di matematica, V. XXV, Fasc. II. 
Zerrik, E., Bourray, H. and Boutoulout, A. (2002). Regional boundary observability, numerical approach, International Journal of Applied Mathematics and Computer Science 12(2): 143-151.

Zerrik, E., Ben Hadid, S. and Bourray, H. (2007). Sensors and regional observability of hyperbolic systems, Sensors and Actuator Journal 138(2): 313-328.

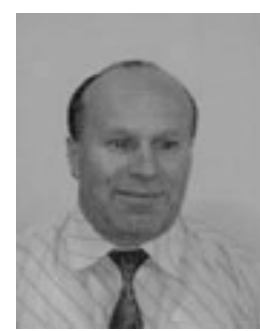

El Hassan Zerrik is a professor at the University of Moulay Ismail of Meknes in Morocco. He has been an assistant professor at the Faculty of Sciences of Meknes and a researcher at the University of Perpignan (France). He obtained his doctorat d' état in systems regional analysis (1993) at the University of Mohammed V of Rabat, Morocco. Professor Zerrik has published many papers in the area of systems analysis and control. Now he is the head of the research team MACS (Modelling Analysis and Control of Systems) at the University of Moulay Ismail of Meknes in Morocco.

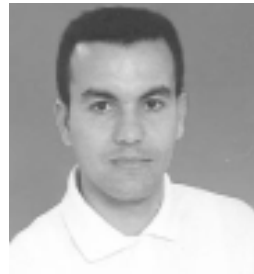

Hamid Bourray is a researcher at the University Moulay Ismail of Meknes in Morocco. He obtained his doctorate in systems analysis and control (2002) at the same university. Dr. Bourray has published many papers in the area of systems analysis and control. At present he is a professor at the Faculty of Poly Disciplinary of Errachidia and a researcher in the group on Methods of Analysis and Control of Systems (MACS) at the University of Moulay Ismail, Meknes, Morocco.

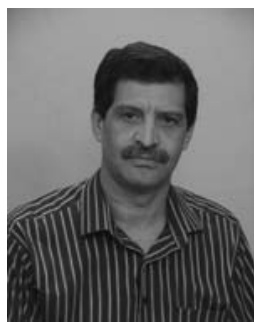

Samir Ben Hadid is a professor at the Mentouri University of Constantine in Algeria. He was awarded his Master's in numerical analysis at the Faculty of Exact Sciences in Constantine in 1997. $\mathrm{He}$ is also a researcher on the MACS team in Morocco and at the laboratory of simulation at the Mentouri University of Constantine in Algeria.

Received: 15 February 2009

Revised: 22 October 2009 